\title{
Towards a Sensorless Current and Temperature Monitoring in MOSFET-Based H-Bridge
}

Buttay Cyril, Bergogne Dominique, Morel Hervé, Allard Bruno, IEEE member, Ehlinger rené and Bevilacqua Pascal

CEGELY-INSA, Bât. Léonard de vinci, Av. Capelle ouest, 69621 Lyon, France

\begin{abstract}
Chip temperature in power MOSFETs is commonly obtained (e.g. for thermal characterisation purposes) by feeding a known, controlled low DC-current in the body diode and measuring the forward-voltage drop.

Drain current might be estimated by a voltage measurement across drain and source terminals, on-state resistance acting as a shunt, assuming that the temperature is known and constant.

Such voltage-based measurements are attractive, but dependencies limit their application to laboratory environment. In industrial converters, neither temperature nor current are known a priori.

Here we show that by combining the two techniques, previous limitations are overcome. Temperature and current come as two separate polynomial expressions.

The application to a low-cost monitoring system is presented and results are discussed. The validity of the system over the full temperature and current ranges is verified and a confidence map is given.
\end{abstract}

\section{INTRODUCTION}

Current and Temperature are critical parameters in power converters. The monitoring of their values is at a premium to ensure reliable operation. Extensive work and literature relate techniques based upon an estimator to avoid the cost and pitfalls of dedicated sensors [1-3]. The method proposed in this paper is based on the measurement of the drain source voltage at the terminals of one MOSFET in an inverter.

Two operating states of the transistor are used to compute current and temperature values. When in the on-state, a MOSFET behaves as a temperature-dependent resistor. Drainto-source voltage is therefore related to the transistor temperature and drain current. When freewheeling on the body diode, the drain-to-source voltage is equal to the intrinsic body diode forward-voltage. This value is mainly governed by the forward (drain) current and the transistor temperature. Thanks to the proximity of the body diode and the channel (a few $\mu \mathrm{m})$, the temperature can be assumed to be identical in both areas. Providing there is a relation between the two currents in the two states, it is possible to extract the temperature and current values.

In this paper, we consider that temperature is constant in the active area of the silicon die. It is referred to as "chip temperature". This is commonly admitted in all circuitsimulation models where device behaviour is described by a set of equations. Further more, the only observable variables in a converter are global such as drain current and drain to source voltage. Taking into account the cellular structure of MOSFET requires the knowledge of difficult to obtain technological parameters. The sophisticated modelisation that would be required is not compatible with a converter

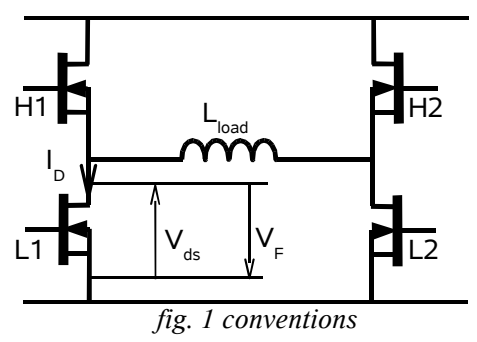

operating in real-time.

\section{PRINCIPLE OF OPERATION}

The two parameters used to compute the temperature and the current have opposite behaviour : $\mathrm{R}_{\mathrm{DSon}}$ presents a positive temperature coefficient whilst the body diode forward-voltage shows a negative temperature coefficient. This allows for a robust discrimination.

In a classical inverter, a deadtime is observed between the turn-off of a pair of switches (e.g. H1 and L2, as described on fig. 1) and the turn-on of the opposite pair (i.e. H2 and L1). Given the load is inductive, current flows through body diodes of $\mathrm{H} 2$ and L1, which will therefore act as freewheeling devices during this period. The forward-voltage of these diodes cannot however be measured at this time, due to the influence of transient phenomena (recovery, overvoltages caused by circuit stray inductances...). To achieve high efficiency, the deadtime must be as short as possible to reduce

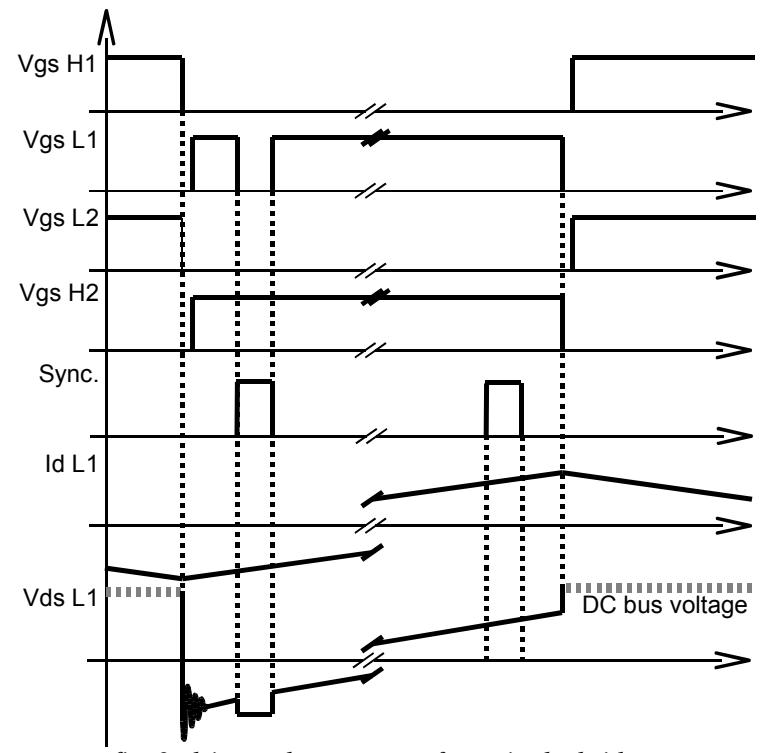

fig. 2. drive and power waveforms in the bridge 
the voltage drop across the body diode, so it doesn't last as long as the transients occurring at commutation. Forwardvoltage will therefore be measured by turning-off the transistor L1 a short lapse of time after the beginning of its conduction. This will not disturb the operation of the inverter, as it doesn't involve a high voltage step (less than 1V) nor high current gradient (the only change in the current path will be across the silicon die). This turning-off must be performed before the current $I_{D}$ becomes positive in the switch (with respect to the conventions above), otherwise it would flow through the diodes of $\mathrm{H} 1$ and L2.

The measurement of the drain-to-source voltage (in the resistive on-state) will be performed just before the end of the conduction, to ensure a good signal-to-noise ratio (the current is maximal at the end of the conduction cycle).

\section{Analytical APPROACH}

\section{A. On-state resistance model}

The effect of the temperature on the on-state MOSFET resistance, is quite well taken into account by classical models such as those implemented in SPICE.

For the sake of simplicity, the equations given here are those of the SPICE level 1 MOS model [4].

In the linear region, i.e. $\mathrm{V}_{\mathrm{GS}}>\mathrm{V}_{\mathrm{TH}}$ and $\mathrm{V}_{\mathrm{DS}}<\mathrm{V}_{\mathrm{GS}}-\mathrm{V}_{\mathrm{TH}}$,

$I_{D}=K P \frac{W}{L-2 X_{j l}}\left(V_{G S}-V_{T H}-\frac{V_{D S}}{2}\right) V_{D S}\left(1+\lambda V_{D S}\right)$

Where KP is the transconductance parameter, $\mathrm{W}$ and $\mathrm{L}$ respectively the width and length of the channel, $X_{j 1}$ the metallurgical junction depth, $\mathrm{V}_{\mathrm{GS}}$ the gate to source voltage, $\mathrm{V}_{\mathrm{TH}}$ the gate to source threshold voltage, and $\lambda$ is the channel length modulation.

For power MOSFETs, $\lambda$ is considered as zero. KP can be supposed to be the only temperature dependent parameter,

$$
K P\left(T_{2}\right)=K P\left(T_{1}\right)\left(\frac{T_{1}}{T_{2}}\right)^{\frac{3}{2}}
$$

The influence of the temperature on the on-state resistance is given by replacing (2) into (1), and then deriving.

$$
\frac{\partial I_{D}}{\partial V_{D S}}=K P\left(T_{1}\right)\left(\frac{T_{1}}{T_{2}}\right)^{\frac{3}{2}} \frac{W}{L-2 X_{j l}}\left(V_{G S}-V_{T H}-V_{D S}\right)
$$

Notice that $\mathrm{R}_{\mathrm{DSon}}$ evolves as a power of 1.5 of the temperature. On a limited temperature range, (3) can therefore be approached by the $2^{\text {nd }}$ order polynomial below.

$$
R_{D S o n}=C_{0}+C_{1} T+C_{2} T^{2}
$$

\section{B. Intrinsic body diode forward-voltage model}

As it can be seen in the extensive literature [5-7], there are few simple but accurate formulations of the diode forwardvoltage drop. This is due to the great number of phenomena occurring in a classical PIN diode. Ohmic contacts are of a major concern in this voltage drop, although they are hardly taken into account in numerous models. The temperature and doping gradients across the $\mathrm{PN}$ junction have also an influence, but are difficult to quantify.

In [5], an analytical expression is used to determine the junction temperature of a power diode from its forwardvoltage and current, but is far too complex for the targeted application.

Thought for monitoring purposes, the proposed method is not supposed to return very accurate estimations. Unavailable technological parameters of the diode prevent the building of a physical model. An empiric model is proposed in (5), its validity will be studied later in this paper. Notice that special care is taken in the fitting of such simple model in order to remain accurate where necessary.

$$
V_{F}=V_{S}(T)+K_{2} I
$$

$\mathrm{V}_{\mathrm{s}}(\mathrm{T})$ is the temperature dependant diode threshold voltage, and $\mathrm{K}_{1}$ a fixed coefficient.

$V_{S}=K_{0}+K_{1} T$

Therefore

$V_{F}=K_{0}+K_{1} T+K_{2} I$

\section{Temperature and current expressions}

From (4) it comes, when in the on-state:

$$
\begin{aligned}
& V_{D S 1}=R_{D S o n} I \\
& V_{D S 1}=\left(C_{0}+C_{1} T+C_{2} T^{2}\right) I_{1}
\end{aligned}
$$

when in the "freewheeling" mode, (7) yields to

$$
V_{D S 2}=-V_{F}=-K_{0}-K_{1} T-K_{2} I_{2}
$$

$\mathrm{V}_{\mathrm{DS} 1}$ is measured at the beginning of the conduction period, while $\mathrm{V}_{\mathrm{DS} 2}$ is the value of the drain-to-source voltage at the end of the conduction. $\mathrm{I}_{1}$ and $\mathrm{I}_{2}$ are therefore different, but $\mathrm{T}$ can be considered as constant, due to the high thermal time constant (Thermal constants are higher than $1 \mathrm{~ms}$ while conduction period in our case is $25 \mu \mathrm{s}$ ).

When using a pure inductive load, $\mathrm{I}_{1}$ and $\mathrm{I}_{2}$ are linked by: 


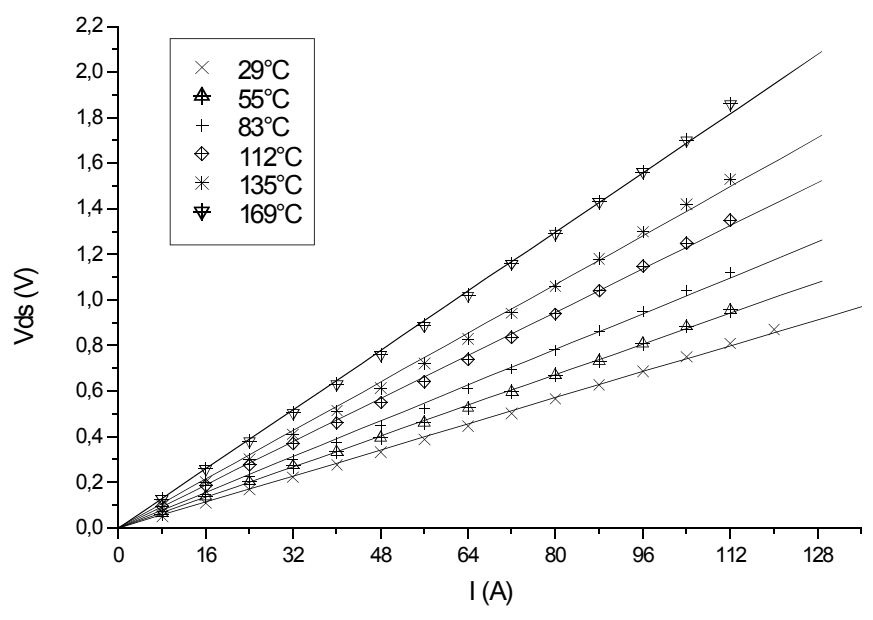

fig. 3. On-state voltage drop for several ambient temperatures

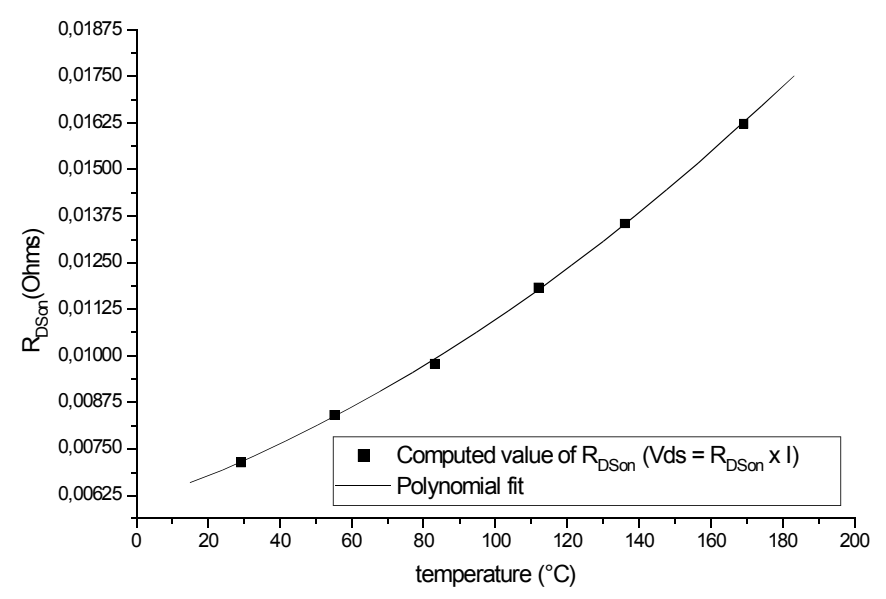

fig. 4. Evolution of the MOSFET on-state resistance as a function of temperature

$$
I_{2}=I_{1}-\frac{U \Delta t}{L_{\text {load }}}
$$

where $\Delta t$ is the time interval between acquisition of $V_{D S o n}$ and acquisition of $\mathrm{V}_{\mathrm{F}}, \mathrm{U}$ is the DC-bus voltage, and $\mathrm{L}_{\text {load }}$ the load inductance. From (9), it comes:

$$
I_{2}=-\frac{V_{D S 2}+K_{0}+K_{1} T}{K_{2}}
$$

Using (10) and (11) in (8) yields:

$$
V_{D S 1}=\left(C_{0}+C_{1} T+C_{2} T^{2}\right)\left(\frac{U \Delta t}{L_{\text {load }}}-\frac{V_{D S 2}+K_{0}+K_{1} T}{K_{2}}\right)
$$

As all parameters $\left(\mathrm{C}_{0}, \mathrm{C}_{1}, \mathrm{C}_{2}, \mathrm{~K}_{.0}, \mathrm{~K}_{1}, \mathrm{~K}_{2}, \mathrm{~L}_{\text {load }}\right)$ are constant and $\Delta t, V_{D S 1}, V_{D S 2}$ are measured, $T$ is obtained by solving the third order polynomial (12). Equation (11) gives the corresponding $\mathrm{I}_{2}$ value.

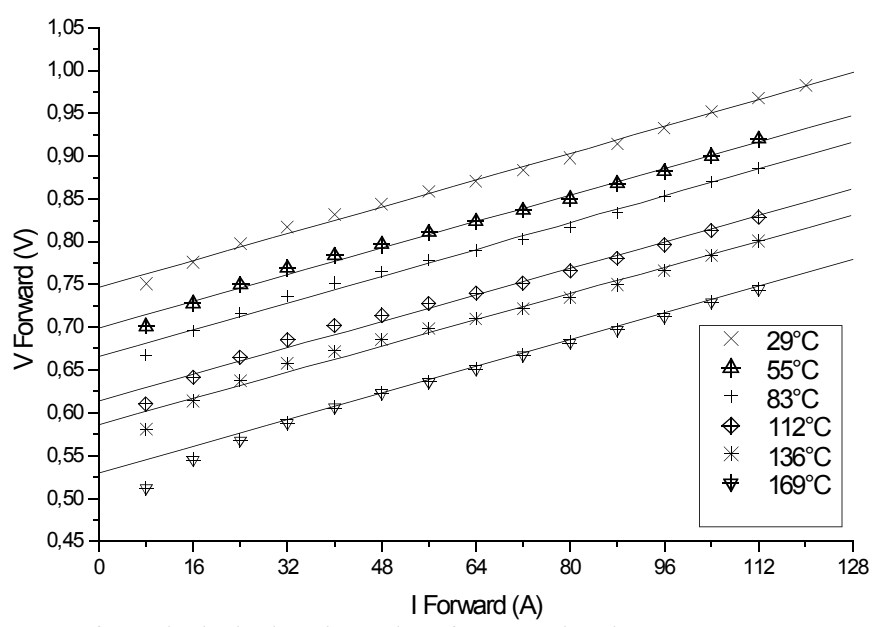

fig. 5. body diode voltage drop for several ambient temperatures

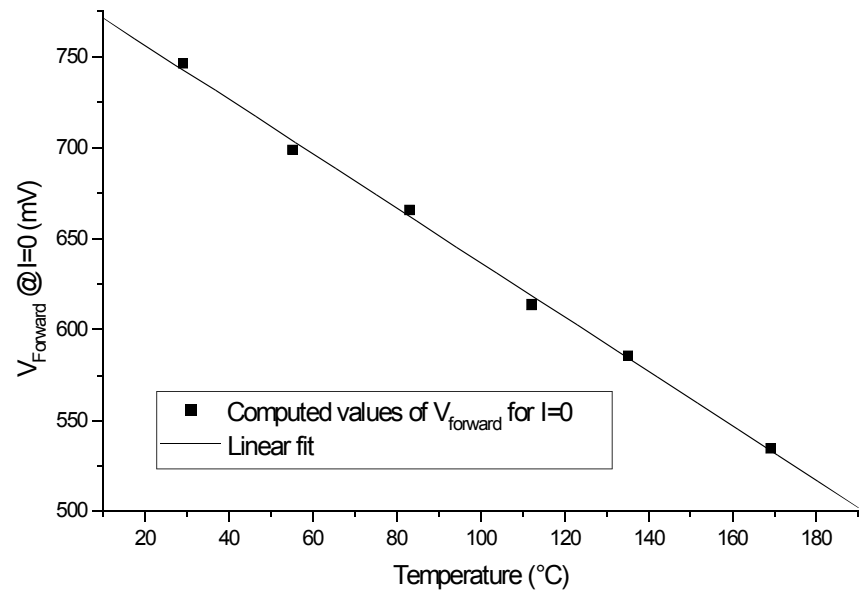

fig. 6. Evolution of the MOSFET body diode threshold voltage as a function of temperature

\section{Parameter estimation}

\section{A. Description of the test bench}

In order to characterise the studied MOSFET (75V 100A), an $\mathrm{H}$ bridge is built using two industrial inverter legs. It is placed in a controlled temperature furnace. To achieve good thermal spreading, an insulated metal substrate (I.M.S) mounted on a heatsink was used. Burst square signals are used to drive the transistors, avoiding self-heating (see fig. 9). However, slight self-heating can be observed at the end of the conduction period (see fig. 8), with the increase in $\mathrm{R}_{\mathrm{DSon}}$. It has little effects on the voltages measured during the parameter estimation, and is taken into account in the fitting.

A small load is required in order to reach the full current during one single period. This imposes an experimental condition where both measurements $\left(\mathrm{V}_{\mathrm{DS}}\right.$ and $\left.\mathrm{V}_{\mathrm{F}}\right)$ occur within a short period of time, thus avoiding temperature variations. Placing the inverter in the furnace has a detrimental effect on load current linearity (see fig. 7) as the electrolytic capacitors bank is kept out of the furnace (electrolytic capacitors cannot 


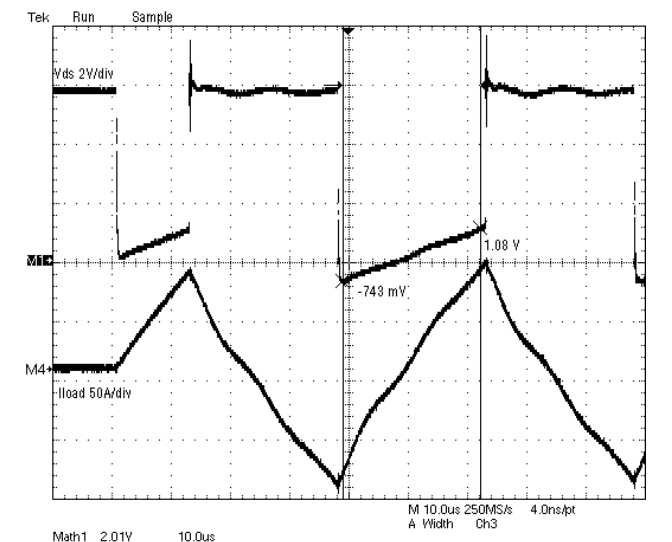

fig. 7 Clamped drain-to-source voltage and corresponding drain current measured by a dedicated probe, showing voltage measurements used for the current and the temperature estimation

withstand $180^{\circ} \mathrm{C}$ ) resulting in a non-negligible stay inductance along the voltage supply conductors.

The drain-to-source voltage is acquired using two P6139 $500 \mathrm{MHz}$ passive probes in a differential setup and a TDS7054 Tektronix digital oscilloscope. Current is measured with a Pearson Electronics model 101, $80 \mathrm{MHz}$ bandwidth current transformer. Drain-to-ground and source-to-ground voltages were clamped using Zener diodes to avoid saturation of the oscilloscope input amplifiers.

Temperature is acquired as the average value given by two thermocouples put at both end of the bridge heatsink. Temperature value is therefore accurate within the $\pm 1^{\circ} \mathrm{C}$ range. More accuracy would be very difficult to achieve, due to the size of the active area (active area of the silicon MOS die). Higher accuracy is not required because of the simplicity of the proposed model for the expression of the body diode forward voltage drop.

Fig. 3 represents the measured drain-to-source voltage when the MOSFET is in the on-state, for 6 different ambient temperatures. As can be seen on fig. 4 , the $\mathrm{R}_{\mathrm{DSon}}$ evolves as a $2^{\text {nd }}$ order polynomial:

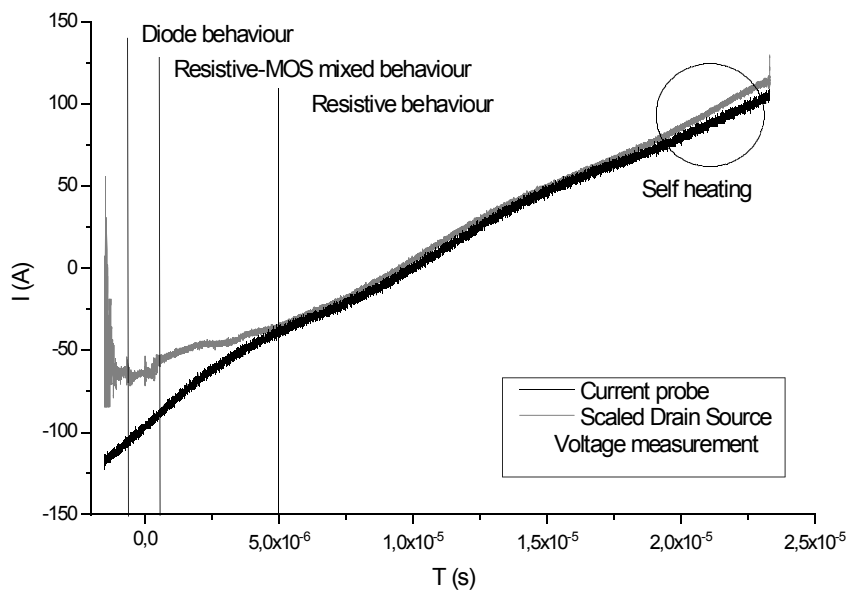

fig. 8 Drain to source voltage during the conduction period.

$$
R_{D S o n}=C_{0}+C_{1} T+C_{2} T^{2}
$$

When fitting data, such a polynomial yields to:

$$
\begin{gathered}
\mathrm{C}_{2}=16 \times 10^{-8} \Omega . \mathrm{T}^{-2} \\
\mathrm{C}_{1}=32 \times 10^{-6} \Omega . \mathrm{T}^{-1}, \\
\mathrm{C}_{0}=58 \times 10^{-4} \Omega
\end{gathered}
$$

The body diode forward-voltage, shown on fig. 5 is more difficult to identify. Fitting is set to minimise error at high current, near SOA (Safe Operation Area) limits. Error is therefore not negligible at low current. Higher order modelling would be required to reduce this inaccuracy, but is beyond of the scope of the targeted application.

In our case, a simple expression gives the value of the body diode voltage drop:

$$
V_{F}=V_{S}(T)+K_{2} I
$$

The relation between Vs and temperature can be seen on fig. 6 , and is expressed by (6). Body diode forward voltage can therefore be expressed as follow:

$$
V_{F}=K_{0}+K_{1} T+K_{2} I
$$

From the identification of data with (7) it comes:

$$
\begin{gathered}
\mathrm{K}_{2}=1.9 \times 10^{-3} \Omega \\
\mathrm{K}_{1}=-1.5 \times 10^{-3} \mathrm{~V} . \mathrm{K}^{-1} \\
\mathrm{~K}_{0}=0.786 \mathrm{~V}
\end{gathered}
$$

This set of parameters $\left(\mathrm{C}_{0}, \mathrm{C}_{1}, \mathrm{C}_{2}, \mathrm{~K}_{0}, \mathrm{~K}_{1}, \mathrm{~K}_{2}\right)$, allows therefore for the calculation of temperature and current across the MOSFET by only proceeding to two distinct drain-source voltage measurements.

Solving (12) for the measurements performed on fig. 7, where $\mathrm{V}_{\mathrm{DS} 1}=1.08 \mathrm{~V}$ and $\mathrm{V}_{\mathrm{DS} 2}=-0.743 \mathrm{~V}$ and the parameters computed above, gives $\mathrm{T}=133^{\circ} \mathrm{C}$ and $\mathrm{I}_{2}=84 \mathrm{~A}$, while the temperature imposed by the furnace was $128^{\circ} \mathrm{C}$ and the

current at the time of the acquisition is $88 \mathrm{~A}$.

\section{Application and Discussion}

It has been established in the previous section the possibility to compute the temperature and current in any MOSFET of the H-bridge by performing two voltage measurements across the device.

In this section, an implementation of the method is described, and the results are discussed.

A monitoring system is built around the H-Bridge: a differential amplifier senses the voltage across the MOSFET for a fast sample-and-hold stage. An analog-to-digital conversion and a data processing is devoted to a small outline microcontroller. One can notice that both analog data (diode forward-voltage drop and on-state MOSFET voltage) use the same acquisition path.

The previous equations are coded into the microcontroller which displays temperature and current values concerning the monitored MOSFET. 


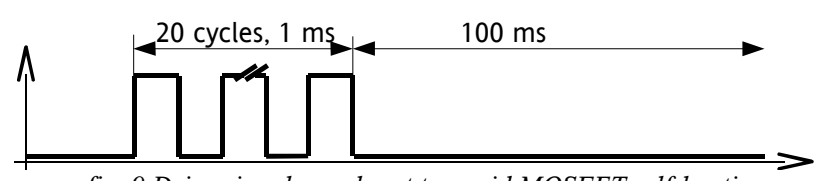

fig. 9 Drive signals are burst to avoid MOSFET self-heating

The analytical approach is chosen for its advantages: ease of calibration (device characteristics dispersion is taken into account by updating a six parameters only), small memory requirement (an equation set replaces a measurement look-up table).

With respect to our application, some assumptions can be made: Load current is pure AC triangular-shaped. We also have identity of the duration between the beginning of L1 conduction and $\mathrm{V}_{\mathrm{F}}$ measurement, and duration between $\mathrm{V}_{\mathrm{DSon}}$ measurement and turning-off of L1. So we may assume $\mathrm{I}_{1}=-\mathrm{I}_{2}$. It comes from (12):

$$
V_{D S 1}=-\left(C_{0}+C_{1} T+C_{2} T^{2}\right) \frac{V_{D S 2}+K_{0}+K_{1} T}{K_{2}}
$$

\section{A. Results}

To solve the previous equation, a dichotomy algorithm is used. It can be seen on fig. 10 that over the whole shaded area the Temperature is monotonous with respect to $V_{f}$ and $V_{D S}$, and will not therefore "trap" the algorithm. Current value is then directly calculated from (11).

As the current waveform has a fixed shape, the system can directly display the RMS current value. This allows for precise measurement using the oscilloscope, as it integrates current value over several periods. Peak and RMS current values are simply proportional in a $\sqrt{3}$ ratio.

Temperature computation requires about $1 \mathrm{~ms}$ with a general purpose low-outline 8-bit microcontroller running at $20 \mathrm{MHz}$. This is due to the intense use of floating point 32-bits numbers, and the dichotomy algorithm which requires 8 solving iterations of (12) in the worst case (as temperature is coded as an 8-bits integer). Current computation is a lot faster (few tens of $\mu \mathrm{s}$ ) as it only requires a single floating point

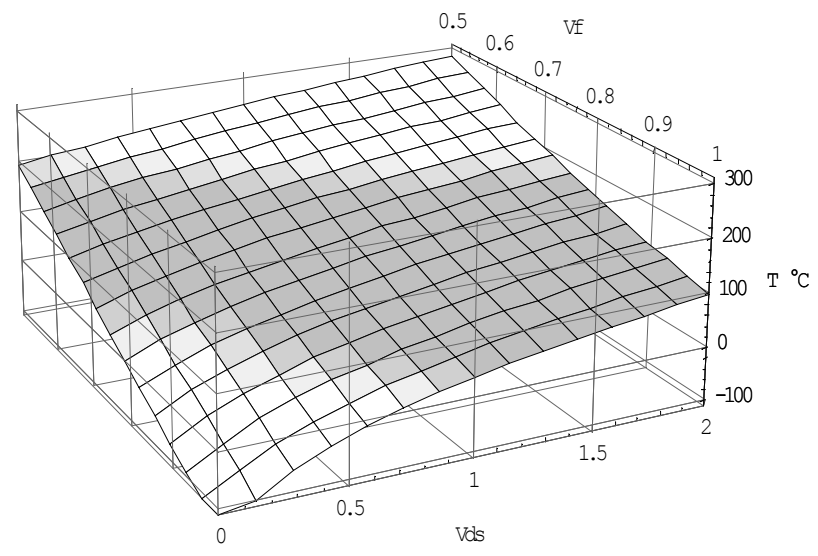

fig. 10. computed temperature over the whole Vds, Vfrange. Shaded area corresponds to operating temperature.

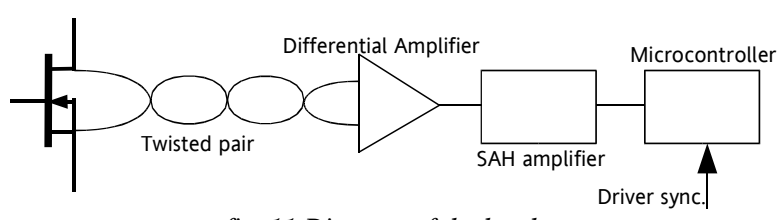

fig. 11 Diagram of the hardware

multiply and two additions.

In order to measure the accuracy of the monitoring system, it is tested over the full current and temperature ranges of the MOSFETs, using previously described test bench where temperature is set using a furnace. These measurement points are chosen different from those of the parameters estimation (above) to ensure our method is reliable. Driving of the bridge is still made using burst square signals to avoid self-heating that would result in different chip and heatsink temperatures.

The current and temperature estimated by the monitoring system are compared to the values measured by the current probe and the thermometer. For the sake of brevity, a global error factor of merit, combining error values on current and temperature is proposed:

$$
\Delta_{I, T}=\sqrt{\Delta_{I}^{2}+\Delta_{T}^{2}}
$$

where

$$
\Delta_{x}=\frac{x_{\text {measured }}-x_{\text {estimated }}}{x_{\text {measured }}}
$$

Fig. 12 Shows the value of $\Delta_{\mathrm{I}, \mathrm{T}}$ over the whole operating range of the MOSFET.

We can see that this monitoring system is reliable, as it computes accurate current and temperature values at high current or high temperature values (near SOA limits).

Error is relatively high at low current level, due to limitations of the estimator: current is calculated as an integer, resulting in high rounding errors under 10 amps. However, the error does never exceed 2 amps over the whole map, and is

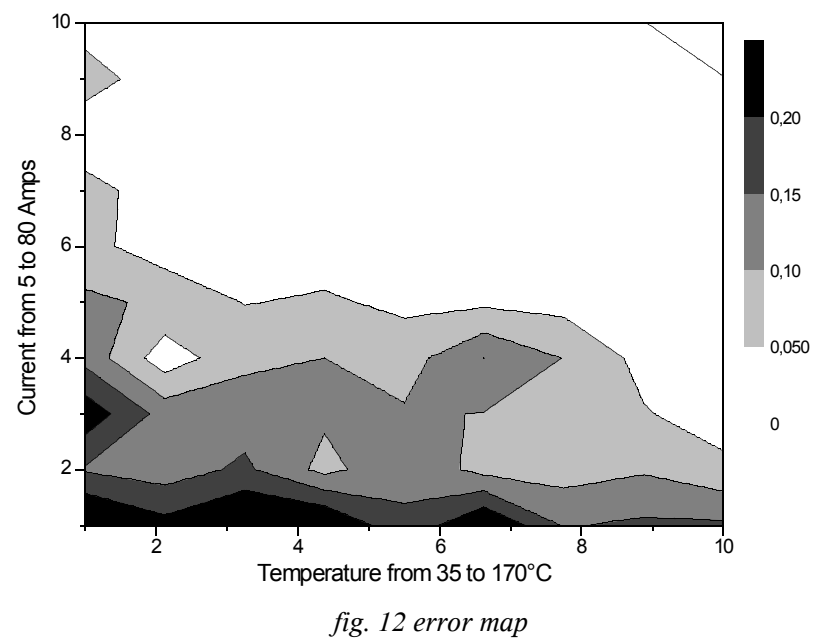

fig. 12 error map 
mainly less than $1 \mathrm{amp}$. This accuracy is achieved thanks to the -relatively- low sensitivity of $V_{D S o n}$ with respect to the temperature.

Another issue at low current level is caused by the assumptions we make on body diode forward voltage drop. The hypothesis of proportionality between body diode forward voltage and drain current is accurate at high current level (see fig. 5), but as current value decreases, the error grows. Fitting of measurement curves is chosen to optimise estimation at high-current (to achieve high reliability), at the cost of worse results when switching low current. This does not conflict with the monitoring purposes, mainly thought for reducing security margins and therefore increasing MOSFET usage.

\section{FURTHER DEVELOPMENTS AND CONCLUSION}

Implementation of the proposed method is described in the previous section. The application is based on two industrial inverter legs connected as a H-Bridge, operating over the full current and temperature range, as specified in device datasheets. However, driving signals (fixed duty cycle) and load (almost pure inductance, with a low value with respect to the switching frequency) are adapted to suit a demonstration purpose only, where the current in one of the switches changes its sign during every commutation cycle. This is not the case of a majority of converters.

Therefore further developments are required to adapt the method for "Real World Applications".

Thanks to current and temperature properties (long time constant vs. switching frequency, periodic behaviour, isothermal areas...) some assumptions can be made, allowing the implementation of the presented technique in operational converter.

Faster estimation of temperature is possible by replacing the 8-bit microcontroller used in our prototype by a low-end signal-oriented processor. Real time computation of the parameters can then be expected. On the other hand, one can use a data look-up table containing current and temperature values corresponding to given $V_{F}$ and $V_{D S}$ instead of the analytical approach described in this paper. The computing power and time required by this method is almost zero, at the extra cost of some kilo-bytes of EPROM.

In addition, it appears that temperature estimation is very sensitive to the forward voltage drop of the body diode. A high resolution measurement of this voltage (such as 14-bit $\mathrm{A} / \mathrm{D}$ conversion) would be required to increase estimation accuracy.

A special interest is the monitoring of paralleled devices in inverters, where transistors are used very intensively, and current sensors are difficult to integrate.

An interesting feature is that the proposed method gives a reliable approximation of the MOSFET die temperature. Classical techniques using external sensor, such as thermistors only give substrate or ambient temperature, which is sometimes quite different from chip temperature, especially during load surges. Accurate knowledge of temperature allows for a better usage of active components by reducing security coefficients, and therefore decreasing overall system cost.

As a conclusion, it is confirmed that the proposed method, based on voltage measurements, allows for the estimation of two key variables in MOSFET-based converters : current and internal chip temperature.

\section{REFERENCES}

[1] D. Bergogne, B. Allard, H. Morel, "An estimation method of the channel temperature of power MOS devices", Proceedings of the PESC'00 conference, Galway, 2000, pp 1594-1599.

[2] B. Allard, H. Morel, K. Ammous, et al., "Application of averaged Models to Real-Time Monitoring of Power Converters", Proceedings of the PESC'01 conference, Vancouver, pp 486 -491, vol.2.

[3] A. Ammous, B. Allard, H. Morel, "Transient Temperature Measurements and Modelling of IGBT's under Short Circuit", IEEE Trans. on Power Electronics, Vol. 13, NO. 1, pp 12-25, 1998

[4] G. Massobrio, P. Antognetti, "Semiconductor device modelling with SPICE", New York: McGraw-Hill, Second edition, 475 p.

[5] F. Profumo, A. Tenconi, S. Facelli, B. Passerini, "instantaneous Junction Temperature Evaluation of High Power Diodes (Thyristors) During Current Transients", IEEE Trans. On Power Electronics, Vol. 14, NO. 2, March 1999, p 292-299

[6] A.Maxim, G. Maxim, D. Andreu, J. Boucher, "Power PIN Diode ElectroThermal SPICE Macromodel with Forward and Reverse Recovery", Proceedings of the EPE'99 conference, Lausanne, 1999.

[7] A. Muñoz-Yagüe, P. Leturcq, "High Level Behavior of Power Rectifiers: A Quantitative Analysis of the Forward Voltage Drop", IEEE Trans. On Electron Devices, Vol. ED25, NO 1, January 1978

[8] B. J. Baliga, "Modern Power Devices", John Wiley \& Sons, 1987

[9] Dr. Martin März, Paul Nance "Thermal Modeling of Power-electronic Systems", Infineon Technologies AG, Munich, available online: http: //www.infineon.com/cmc_upload/

migrated_files/document_files/Application_Notes/mmpn_eng.pdf 